\title{
Cytological sampling at fibreoptic bronchoscopy: comparison of catheter aspirates and brush biopsies
}

\author{
MF MUERS, MM BODDINGTON, MARGARET COLE, D MURPHY, AI SPRIGGS \\ From the Chest Department and Cytology Laboratory, Churchill Hospital, Oxford, and the Cytology \\ Laboratory, Royal Berkshire Hospital, Reading
}

ABSTRACT A prospective study was undertaken to compare bronchial brushings with dry catheter aspiration for the cytological diagnosis of lung cancer at fibreoptic bronchoscopy. Duplicate samples taken by aspirate and brush were obtained at 103 consecutive routine bronchoscopies. Aspirate and brush samples were reported on separately by two cytologists. At the end of the study a $30 \%$ sample, including the 19 cases with different findings by the two methods, were subjected to a blind crossover review, and then an open review. Forceps biopsy specimens for routine histological assessment were taken in 94 cases (92\%). Ninety-eight of $103(95 \%)$ aspirates and 99 of $103(96 \%)$ brush specimens were technically satisfactory. Carcinoma was diagnosed at bronchoscopy on cytological or histological evidence or both in 57 cases. Fifty-five of these tumours $(96 \%)$ were recognised by either the aspirate or the brush method, $52(91 \%)$ by brush, and $50(88 \%)$ by aspirate. Thirty-four cases $(60 \%)$ could be diagnosed from histological specimens. The order of cytological sampling did not systematically affect yield. It is concluded that fine-catheter aspirates, permitting smears to be prepared in the laboratory, are a satisfactory alternative to brush smears for the cytological diagnosis of lung cancer at bronchoscopy. The routine use of both techniques at bronchoscopy will increase diagnostic yield by about $5 \%$. Duplicate sampling may be especially useful in obtaining diagnostic material from upper-lobe or apical-segment tumours.

Since the introduction of fibreoptic bronchoscopy material for cytological diagnosis of lung cancer has generally been obtained by the brushing method. ${ }^{1-3}$ Brush cytology has been shown to have a greater diagnostic yield than the examination of saline bronchial washings or of postbronchoscopy sputum.4-6 Brush samples are sometimes spoilt, however, by low cellularity or by drying before fixation, and an alternative method that has administrative and technical advantages has been described. ${ }^{7}$ This consists of the aspiration of material from suspected areas into a disposable plastic catheter. Portions of catheter containing secretion are cut off and sent for processing at leisure in the laboratory rather than hastily in the bronchoscopy room. This method has been used in the unit at Oxford for four years.

Since bronchial brushing remains the standard method elsewhere, we thought that a formal study of the two sampling methods was needed-firstly, to compare their diagnostic accuracy and, secondly, to

Address for reprint requests: Dr MF Muers, Leeds Chest Clinic, 74 New Briggate, Leds LS1 6PH. assess whether the use of both methods at one examination would increase diagnostic sensitivity.

\section{Methods}

Routine fibreoptic bronchoscopies were performed in our bronchoscopy unit by two of us, both having undertaken more than 150 examinations at the beginning of the study. Examinations were carried out at the request of other physicians, and were performed transnasally under local anaesthesia after premedication with atropine, diazepam, and codeine phosphate. Olympus BFIT and BFB3 bronchofibrescopes were used. Samples were taken from areas that appeared abnormal, or blindly from segments corresponding to radiological opacities if there were no endobronchial abnormalities. Screening was not used. The order of brushing and aspiration was reversed in successive cases, and forceps biopsy samples for histological examination were obtained afterwards unless bleeding prevented this.

Bronchial brushings were obtained with the unsheathed BFIC instrument. Immediately after 
sampling, the instrument was withdrawn from the fibrescope and the brush rubbed firmly with a gloved finger on to a limited area of a microscope slide with a circular motion. Rubbing was limited to five seconds to minimise air-drying artefacts and the slides were then immersed in ethanol fixative in a Coplin jar for transport to the laboratory.

Bronchial aspirates were obtained with disposable polyethylene catheter tubing of $1.5 \mathrm{~mm}$ (outside diameter) (Vygon Ltd). The catheter tip was probed over a lesion while gentle suction was applied to the proximal end by means of a $20-\mathrm{ml}$ syringe and a 21 -gauge needle inserted into the tubing. Suction was applied for about 30 seconds, and then the tubing was withdrawn. The bronchoscopy examination was then completed, and after it lengths of tubing containing aspirated material were cut out and placed in dry screw-topped containers. As it has been found that air drying does not occur in this fine tubing, samples were prepared electively in the laboratory by using a fine pasteur pipette to expel the secretion on to slides for immediate smearing, fixation, and staining.

Duplicate slides were prepared for each patient with each method and were stained by the Papanicolaou technique. Samples for histological examination were fixed in formol saline and assessed routinely in the local histopathological laboratory.

Examination of specimens Firstly, the two cytologists independently examined the specimens after each bronchoscopy session, one in Oxford and the other in Reading. Observer 1 reported on the aspirates and observer 2 on the brushings. They were not aware of each other's reports and the specimens were not reviewed at the time. Both observers were experienced with both types of sample. Secondly, half way through the study and at the end, the initial reports were correlated by the two physicians, who prepared a $30 \%$ sample for blind crossover review. These samples included all the specimens where there were differences between the first reports on the brushings and aspirates, and also included a proportion of samples with agreed diagnoses as controls. Finally, after the blind crossover reviews samples with reporting differences were reviewed again by observers 1 and 2 working together.

\section{Results}

\section{ADEQUACY OF SAMPLES}

Specimens were obtained from 103 bronchoscopies performed on 98 patients. Brushings were taken first on 50 occasions and aspirates first in 53. Of the 206 samples, all but nine $(96 \%)$ were technically satisfactory. One set of brushings was damaged by drying artefact. The other eight specimens, three brushings and five aspirates, contained insufficient cells for diagnosis. Five of these eight samples, two brushings and three aspirates, were taken blind from upper lobes. A diagnosis of carcinoma was established by other means for all three cases in which the brushings were inadequate, but in only two of the five cases in which the aspirates were inadequate. The overall sampling success rates, 100 of $103(97 \%)$ for the brushings and 98 of $103(95 \%)$ for the aspirates, were felt to be sufficiently close to allow direct comparison of the diagnostic sensitivities to be made.

\section{ANALYSIS OF DISCREPANCIES}

Thirty-four pairs of specimens were submitted for blind crossover review to the two cytologists. These comprised: (1) 10 pairs in which carcinoma had been reported in one specimen but not in the other; (2) nine in which there were reported differences about the malignant cell type; (3) five in which one observer had reported "suspicious" cells; (4) 11 pairs of slides (five cases of carcinoma and six normal cases) included as controls as there had been previous agreement between the two separate reports.

Of the 10 pairs of samples where one of the pair had originally been reported as showing carcinoma and the other had not, the blind crossover review and repeat review showed that only two discrepancies were due to reporting error. In both cases the aspirates were technically adequate. This error level represents an internal consistency of reporting of $97 \%$ on the aspirates and of $100 \%$ on the brushings. The eight remaining discrepancies represented genuine differences in the samples, there being three cases of carcinoma diagnosable from the aspirates with genuinely negative brushings and five diagnosable from the brushings with genuinely negative aspirates. Five of these eight successful samples were the first of the pair to be taken, but this does not provide sufficient evidence to say that the order of cytological sampling affects diagnostic yields. Likewise there was no correlation between the site of sampling and the success of one technique versus the other. From the upper lobes one aspirate and two brushings were successful and from the right middle and lower lobes two aspirates and three brushings. Forceps biopsy samples were positive in three of the eight cases-one in which an aspirate was diagnostic and two in which brushings were.

Review of the five cases in which one or other cytologist had originally reported cells "suspicious" (two aspirates and three brushings) confirmed three of these reports as positive and two as negative. All five pairs of samples were from cases of carcinoma diagnosed from other evidence. 
In our reviews we included the nine cases where there was disagreement between the two original reports about the malignant cell type. After a blind crossover review and then a repeat review five of the nine cases were agreed to be squamous in origin; the four others (7\% of all the carcinomas) could not be accurately typed. In the 18 "blind" reports in this group, observer 1 reported oat-cell carcinoma more often than observer 2 (five versus none) whereas observer 2 reported squamous-cell carcinoma more often than observer 1 (13 versus four). At the nonblind review the cytologists reported no preference for a particular preparation in making a diagnosis of cell type in difficult cases.

\section{FINAL DIAGNOSES OF CARCINOMA}

The number of diagnoses of carcinoma finally made by each of the methods used is shown as a Venn

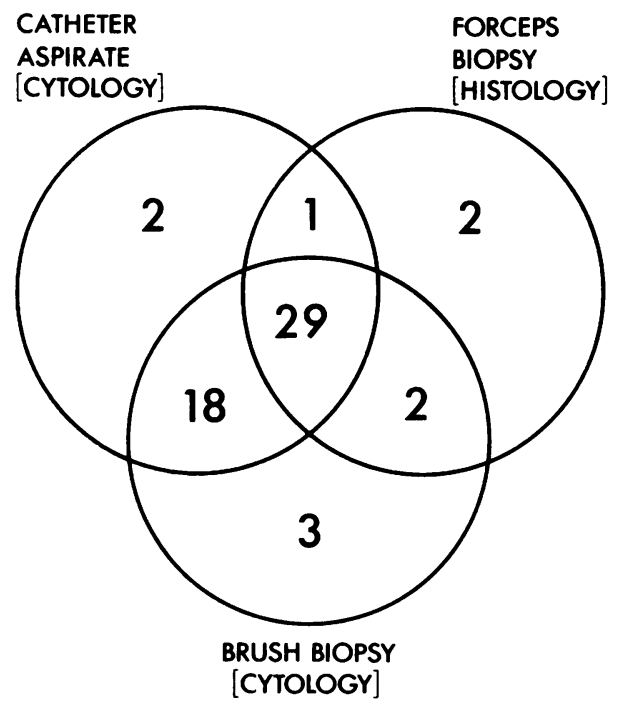

Diagnosis of carcinoma at fibreoptic bronchoscopy analysed by sampling method $(N=57)$.

Analysis of the diagnostic specificity for carcinoma of sampling methods at fibreoptic bronchoscopy

\begin{tabular}{llll}
\hline Method & No positive & $\begin{array}{l}\text { \% of positive } \\
\text { bronchoscopy of final } \\
\text { diagnoses } \\
(N=67)\end{array}$ & $\begin{array}{l}\text { diamours } \\
(N=67)\end{array}$ \\
\hline Catheter and brush & 55 & 96 & 82 \\
Brush and histology & 55 & 96 & 82 \\
Catheter and histology 54 & 95 & 81 \\
Brush alone & 52 & 91 & 78 \\
Catheter alone & 50 & 88 & 75 \\
Biopsy alone & 34 & 60 & 51 \\
\hline
\end{tabular}

diagram in the figure. A morphological diagnosis of malignancy was made at bronchoscopy in 57 of the 103 examinations. The diagnostic yields of the different methods are listed in the table. It is noticeable that both cytological methods alone were superior to forceps biopsy ( $91 \%$ and $88 \%$ versus $60 \%$ ) but that the combination of either method with the results of forceps biopsy increased the sensitivity to $95-96 \%$. The combination of the two cytological methods showed an increase in sensitivity of $8 \%$ (catheter) and $5 \%$ (brush) respectively.

The overall diagnostic sensitivity of fibreoptic bronchoscopy in our series-on the basis of the final diagnosis provided by evidence obtained at bronchoscopy itself or at subsequent thoracotomy or as a result of subsequent clinical investigation and follow-up for at least six months—was 57/67 (85\%). We failed to confirm a diagnosis of carcinoma in 10 cases. In seven cases all tissue samples from an undoubted endobronchial lesion were negative for carcinoma. One of these proved at resection to be an endobronchial asthmatic polyp and another was a small polyp of $2 \mathrm{~mm}^{3}$ that could not be seen at a subsequent rigid bronchoscopy. The other five cases were finally diagnosed as malignant. All had lesions either in the apical segments of the lower lobes (2) or upper lobes (3), and this reflects the well-known technical difficulty of sampling from these areas of the bronchial tree. Catheter and brush sampling were superior in our hands to forceps biopsy in these areas since, of the 19 cases with confirmed malignant lesions, $18(95 \%)$ were diagnosed by cytological techniques but only eight $(42 \%)$ by forceps biopsy.

\section{Discussion}

This study was designed to compare directly two methods of obtaining tissue samples for cytological examination at routine bronchoscopy. For the true difference in diagnostic sensitivity to be assessed, other causes of disparity had to be minimised. The potential sources of error are, firstly, those connected with the acquisition of samples and, secondly, those arising from their interpretation.

We established uniformity of sampling conditions by studying patients prospectively. Both technique and operator skill bias was small as only two experienced bronchoscopists submitted material for examination. We had thought that the order of sampling might have affected yield since even aspiration with the soft catheters sometimes produced appreciable haemorrhage from vascular tumours, but we found that this was not confirmed in practice. Similarly, the $95 \%$ adequacy rate of sampling ensured that differences in the yields of the two techniques 
were not simply reflections of the operators' greater skill with one of them.

The two reviews of the 19 pairs of specimens from patients where there was an initial difference between the reports by the two independent cytologists showed that only two were due to reporting error. Judged therefore by the criterion of internal consistency, the specificity of the reports in this series in assessing the presence of neoplastic cells was $97 \%$.

The second cause of reporting differences not due to sampling technique was the difficulty of ascribing a cell of origin to some tumours. Review enabled an agreed diagnosis to be made in five of the nine cases, but the remaining four tumours were undifferentiated with no characteristic morphological features. The reviews emphasised to us the problems of ascribing a cell of origin to poorly differentiated tumours. This is relevant to comparisons of the relative accuracy of cytological and histological diagnosis at bronchoscopy-for example, the study of Payne $e t a l,{ }^{8}$ who reported a better correlation in the cell of origin diagnosis between sputum cytology and large-specimen histology than between the latter and either forceps biopsy, needle aspiration, or washings. Internal correction of observer bias in difficult cases should even further improve the apparent accuracy of cytological diagnosis in this respect.

In this bronchoscopic study we obtained tissue diagnostic of carcinoma by cytology in $82 \%$ of the 67 cases with final diagnoses of tumour, and positive histological diagnosis in $51 \%$. The sensitivity of these techniques in our series is thus similar to that reported elsewhere, ${ }^{910}$ and evidence is available ${ }^{511}$ to suggest that bronchial brushing is better able to provide diagnostic material at fibreoptic bronchoscopy than is forceps biopsy. We regard these two techniques as complementary, however-though omitting biopsy from our series would have meant a failed diagnosis in only two cases with visible tumour.

In 10 of these 103 bronchoscopies aspirate specimens were not diagnostic (five poor specimens and five missed tumours), compared with six cases where the brush biopsies were unsatisfactory (three poor specimens and three missed tumours). We consider that these differences are too small to allow a value judgment to be made between the two techniques, and certainly this study does not provide evidence that one method is technically superior to the other, provided that both are performed with the necessary care and skill.

Previous studies ${ }^{4-6} 11$ provide ample evidence that bronchial brushing provides a better diagnostic yield than routine bronchial segment washing and postbronchoscopy sputum. Our study suggests that catheter aspiration is a better complementary method to brushing if a routine second sample is to be taken. A second role for catheter aspiration is suggested by the evidence presented here that forceps biopsy of upper-lobe and apical-segment lesions is less likely to give adequate specimens. This suggests that a second cytological sampling from such lesions by catheter would be a useful way of improving diagnostic yield in these difficult cases. It would also be an appropriate method for sampling the different segments in cases with "positive" sputum but without radiological localisation.

In our view it is doubtful whether an improvement in diagnostic yield of $5-8 \%$, which on our evidence would accrue from the routine combination of brushing and aspiration, would justify the doubling of a cytology laboratory's work load. In our unit we have concluded that these two techniques are complementary, and that a second sampling is useful if there is doubt about the size of forceps biopsy specimens. Catheter aspirate samples are in addition very suitable for other investigations, such as microbiological culture, as has been recently reported elsewhere. ${ }^{12}$

Recently Buirski and colleagues ${ }^{13}$ have reported excellent cytological diagnostic accuracy with the use of bronchoscopic needle aspiration. In their hands cytological sampling with this technique had a diagnostic accuracy of $80 \%$ (48 of 60 cases), and this was superior to the results of brushings in the same patients (65\%: 35 of 54 cases). The yield from the brushings, however, was lower than in many other published series as well as our own. ${ }^{2} 611$

Four techniques, however, for tissue sampling at fibreoptic bronchoscopy have now been described-namely, forceps biopsy for histology, and brushing, catheter aspiration, and needle aspiration for cytology. Histological specimens are required in all cases, but bronchoscopists are now faced with a choice of method for complementary sampling. For routine use and where lesions are easily accessible, there seems little to choose between the three methods, and no sufficiently large studies have yet been done to determine whether there is a genuine difference in yield. Pertinent points affecting choice appear to be that, firstly, the catheter apparatus is much cheaper, and rapid slide preparation during an examination is not required; secondly, the brush is easier to manipulate than the catheter and needle, and smears are easier to prepare from it than from the needle; thirdly, for sessile or submucosal lesions that are easily accessible, the needle technique appears best. For upper-lobe lesions which are difficult to sample we recommend that two cytological methods are used in addition to forceps biopsy. For blind sampling from small bron- 
chi, either the brush or the catheter should be used as the aspiration needle has not yet been shown to be safe if used in this way, although haemorrhage does not appear to be a problem when visible lesions are sampled.

We are grateful to the physicians of the chest department at the Churchill Hospital, Oxford, for allowing us to study their patients and also to $J$ Kitaruth at the cytology laboratory, Royal Berkshire Hospital, for his assistance with the slide review.

\section{References}

${ }^{1}$ Ikeda S, Yanai N, Ishikawa S. Flexible bronchofiberscope. Keio J Med 1968;17:1-16.

${ }^{2}$ Zavala D. Diagnostic fiberoptic bronchoscopy. Techniques and results of biopsy in 600 patients. Chest 1975;68:12-9.

${ }^{3}$ Richardson RH, Zavala DC, Mukerjee PK, Bedell GN. The use of fiberoptic bronchoscopy and brush biopsy in the diagnosis of suspected pulmonary malignancy. Am Rev Respir Dis 1974;109:63-6.

4 Solomon DA, Solliday NH, Gracey DR. Cytology in fiberoptic bronchoscopy: comparison of bronchial brushing, washing, and post bronchoscopy sputum. Chest 1974;65:616-9.

${ }^{5}$ Chopra SK, Genovesi MG, Simmons DH, Gothe EB. Fiberoptic bronchoscopy in the diagnosis of lung cancer: comparison of pre- and post-bronchoscopy sputa, washings, brushings, and biopsies. Acta Cytol (Baltimore) 1977;21:524-7.

- Funahashi A, Browne TK, Houser WC, Hranicka LJ. Diagnostic value of bronchial aspirate and post-bronchoscopic sputum in fiberoptic bronchoscopy. Chest 1979;76:514-7.

' Cole Margaret J, Cowie J, Spriggs AI. Disposable polythene catheter: an alternative to the bronchial brushing method for cytology of bronchial secretion obtained with fibreoptic bronchoscope. J Clin Path 1978;31:907-8.

${ }^{8}$ Payne CR, Stovin PG, Barker V, McVittie S, Stark JE. Diagnostic accuracy of cytology and biopsy in primary bronchial carcinoma. Thorax 1979;34:294-9.

${ }^{9}$ Mitchell DM, Emerson CJ, Collyer J, Collins JV. Fibreoptic bronchoscopy: ten years on. $\mathrm{Br} \mathrm{Med} J$ 1980;3:360-3.

${ }^{10}$ Lyall JRW, Summers GD, O'Brien IM, Bateman NT, Pike CP, Braimbridge MV. Sequential brush biopsy and conventional biopsy: direct comparison of diagnostic sensitivity in lung malignancy. Thorax 1980;35:929-31.

" Kvale PA, Bode FR, Kim S. Diagnostic accuracy in lung cancer. Comparison of technique used in association with flexible fiberoptic bronchoscopy. Chest 1976;69:752-7.

${ }^{12}$ Tobin JO'H, Beare J, Dunnill MS, Fisher-Hoch S, French M, Mitchell RG, Morris PJ, Muers MF. Legionnaires' disease in a transplant unit: isolation of the causative agent from shower baths. Lancet 1980;ii:118-21.

${ }^{13}$ Buirski G, Calverley PMA, Douglas NJ, Lamb D, McIntyre M, Sudlow $\mathbf{M}$, White $\mathbf{H}$. Bronchial needle aspiration in the diagnosis of bronchial carcinoma. Thorax 1981;36:508-11. 